\title{
ДВИЖЕНИЕ И ПЕРЕНОС ТЕПЛА В ТУРБУЛЕНТНЫХ ВИХРЕВЫХ КОЛЬЦАХ
}

При действии в ограниченной области заполненного жидкостью пространства мгновенного источника импульса и тепла (или плавучести) возникает движение с характерной для вихревого кольца структурой. Знание этого явления необходимо для решения таких прикладных задач, как развитие грибовидного облака от взрыва мощного заряда или от извержения вулкана, прерывистое удаление в атмосферу дымовых газов, создание искусственных осадков, развитие в атмосфере изолированных конвективных масс, приводящих к образованию кучевых облаков и т. д.

\section{Постановка задачи}

Движение и перенос тепла в вихревом кольце, развивающемся в безграничной несжимаемой жидкости, в случае полностью развитого турбулентного движения могут быть описаны следующей системой дифференциальных уравнений для осредненных параметров течения (при условии справедливости аппроксимации Буссинека и симметрии процесса относительно оси, совпадающей с направлением основного движения вихревого кольца):

$$
\begin{gathered}
\frac{\partial \zeta}{\partial t}+u \frac{\partial \zeta}{\partial x}+v \frac{\partial \zeta}{\partial r}-\frac{v \zeta}{r}=-\beta g \frac{\partial T}{\partial r}+\frac{\partial\left(\overline{-u^{\prime} \zeta^{\prime}}\right)}{\partial x}+\frac{\partial\left(\overline{-v^{\prime} \zeta^{\prime}}\right)}{\partial r} \\
\frac{\partial^{2} \psi}{\partial x^{2}}+\frac{\partial^{2} \psi}{\partial r^{2}}-\frac{1}{r} \frac{\partial \psi}{\partial r}=-r \zeta \quad\left(\zeta=\frac{\partial v}{\partial x}-\frac{\partial u}{\partial r}\right) \\
u=\frac{1}{r} \frac{\partial \psi}{\partial r}, \quad v=-\frac{1}{r} \frac{\partial \psi}{\partial x}, \\
\frac{\partial T}{\partial t}+u \frac{\partial T}{\partial x}+v \frac{\partial T}{\partial r}=\frac{\partial \overline{\left(-u^{\prime} T^{\prime}\right)}}{\partial x}+\frac{\partial \overline{\left(-v^{\prime} T^{\prime}\right)}}{\partial r}-\frac{\overline{v^{\prime} T^{\prime}}}{r}
\end{gathered}
$$

при граничных условиях

$$
\begin{array}{ll}
\psi=\zeta=\frac{\partial T}{\partial r}=0 & (r=0), \\
\psi=\zeta=T=0 & \left(x^{2}+r^{2} \rightarrow \infty\right) .
\end{array}
$$

Необходимые для полного определения задачи интегральные условия однозначности получаются в результате интегрирования (1) и (4) по всей рассматриваемой области течения, т. е. 


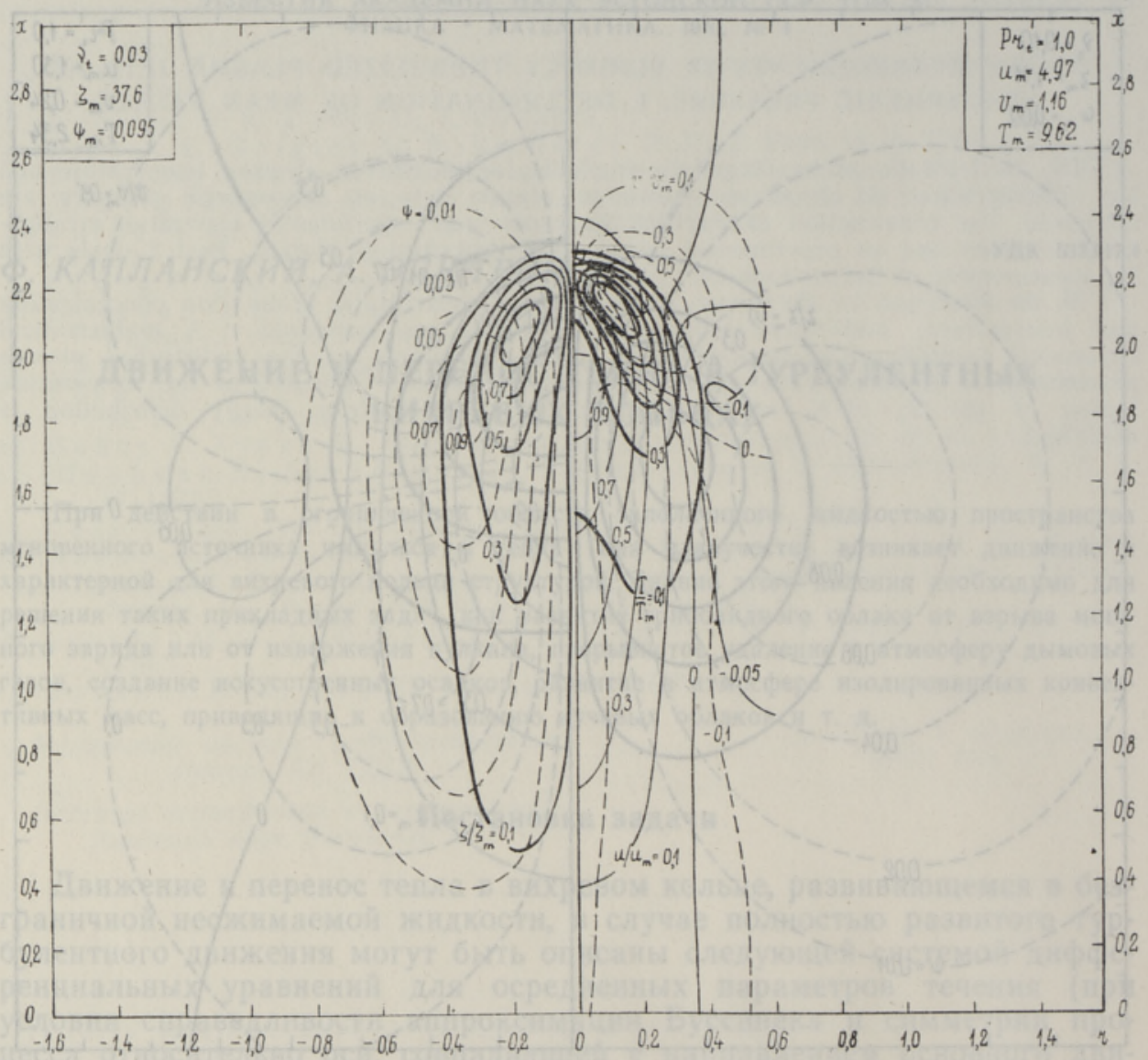

Рис. 2.

плоского аналога вихревого кольца от мгновенного источника тепла (конвективное вихревое кольцо или термик) - в $\left[{ }^{4}\right]$ (см. также $\left.\left[{ }^{5}\right]\right)$. Введем новые переменные $\quad x_{*}=x / L, r *=r / L, u_{*}=u / V, v_{*}=v / V$, $\psi_{*}=\psi / L V, \quad \zeta_{*}=\zeta V / L, \quad T_{*}=T / \Theta$, а также $v_{t^{*}}=v_{t} / L V, a_{t^{*}}=a_{t} / L V$. Заменив переменные в (6а) и (6б) и обозначив

$$
\pi \int_{-\infty}^{\infty} \int_{0}^{\infty} \zeta r^{2} d x d r=\chi_{1}, \quad 2 \pi \int_{-\infty}^{\infty} \int_{0}^{\infty} T r d x d r=\chi_{2}
$$

(здесь и ниже индекс (*) при новых переменных опущен), а также положив $V=x \frac{d L}{d t}$, получим следующие уравнения связи между масштабами

$$
\begin{aligned}
L^{3} \frac{d L}{d t} & =\frac{1}{x \chi_{1}}\left(I_{0}+Q_{0} t\right), \\
L^{3} \Theta & =\frac{Q_{0}}{\chi_{2} \beta g}=\frac{Q_{T 0}}{\chi_{2}} .
\end{aligned}
$$




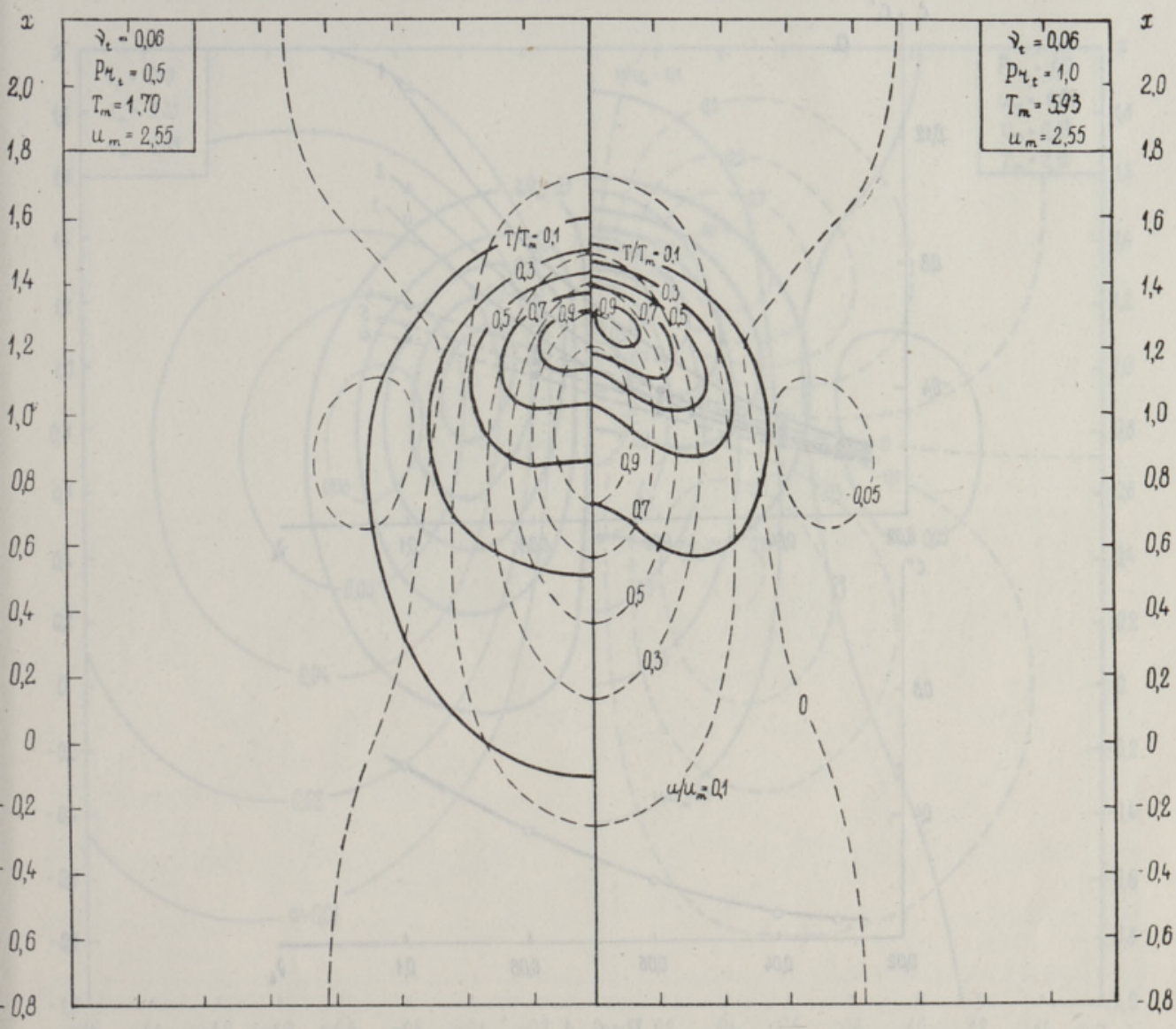

$\begin{array}{lllllllllllllllll}1,6 & -1,4 & -1,2 & -1,0 & -0,8 & -0,6 & -0,4 & -0,2 & 0 & 0,2 & 0,4 & 0,6 & 0,8 & 1,0 & 1,2 & 1,4 & r\end{array}$

Рис. 3.

Рассматриваемая задача является автомодельной в следующих частных случаях:

1) $I_{0} \neq 0, Q_{0}=0$, т. е. для динамического вихревого кольца. Заметим, что при этом можно считать $Q_{T 0} \neq 0$, так как тепловая задача становится здесь независимой от динамической и условие $Q_{0}=0$ достигается за счет $\frac{\beta g L \Theta}{V^{2}} \approx 0$.

2) $I_{0}=0, Q_{0} \neq 0$, т. е. для конвективного вихревого кольца.

Решая (9а) и (9б) при начальном условии $L\left(t_{0}\right)=L_{0}$, для динамического вихревого кольца при выборе $t_{0}=\frac{x \chi_{1} L_{0}^{4}}{4 I_{0}}$ получаем

$$
L=\left[\frac{4 I_{0} t}{x \chi_{1}}\right]^{1 / 4}, \quad V=\frac{1}{4} x_{1}\left[\frac{4 I_{0}}{x \chi_{1}}\right]^{1 / 4} t^{-3 / 4}, \quad \Theta=\frac{Q_{T 0}}{\chi_{2}}\left[\frac{4 I_{0} t}{x \chi_{1}}\right]^{-3 / 4}
$$

и для конвективного вихревого кольца при $t_{0}=\left[\frac{x \chi_{1} L_{0}^{4}}{2 Q_{0}}\right]^{1 / 2}-$ 


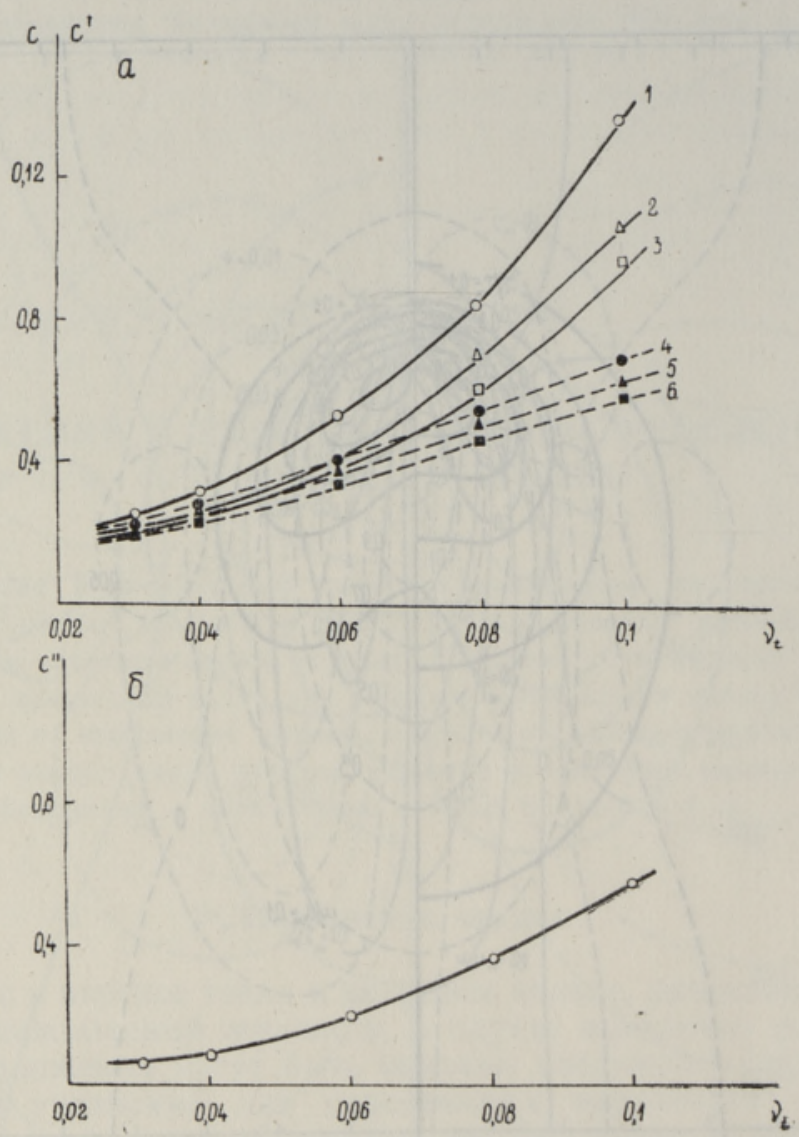

Рис. 4.

$L=\left[\frac{2 Q_{0} t^{2}}{x \chi_{1}}\right]^{1 / 4}, \quad V=\frac{1}{2} x\left[\frac{2 Q_{0}}{x \chi_{1}}\right]^{1 / 4} t^{-1 / 2}, \quad \Theta=\frac{Q_{0}^{1 / 4}}{\chi_{2} \beta g}\left[\frac{2}{x \chi_{1}}\right]^{-3 / 4} t^{-3 / 2}$

Коэффициенты $\chi, \chi_{1}$ и $\chi_{2}$ можно рассматривать здесь как произвольные константы, значения которых выбираются из соображений удобства при численном решении задачи.

В результате соответствующих преобразований с учетом (10) или (11), а также (7), уравнения (1) и (4) в безразмерных переменных принимают следующий вид:

$$
\begin{gathered}
\frac{\partial \zeta}{\partial \tau}-A \zeta+(x u-x) \frac{\partial \zeta}{\partial x}+(x v-r) \frac{\partial \zeta}{\partial r}-\frac{x v \zeta}{r}= \\
=-B \frac{\partial T}{\partial r}+x v_{t}\left[\frac{\partial^{2 \zeta}}{\partial x^{2}}+\frac{\partial^{2 \zeta}}{\partial r^{2}}+\frac{\partial}{\partial r}\left(\frac{\zeta}{r}\right)\right] \\
\frac{\partial T}{\partial \tau}-C T+(x u-x) \frac{\partial T}{\partial x}+(x v-r) \frac{\partial T}{\partial r}= \\
=x a_{t}\left(\frac{\partial^{2} T}{\partial x^{2}}+\frac{\partial^{2} T}{\partial r^{2}}+\frac{1}{r} \frac{\partial T}{\partial r}\right)
\end{gathered}
$$




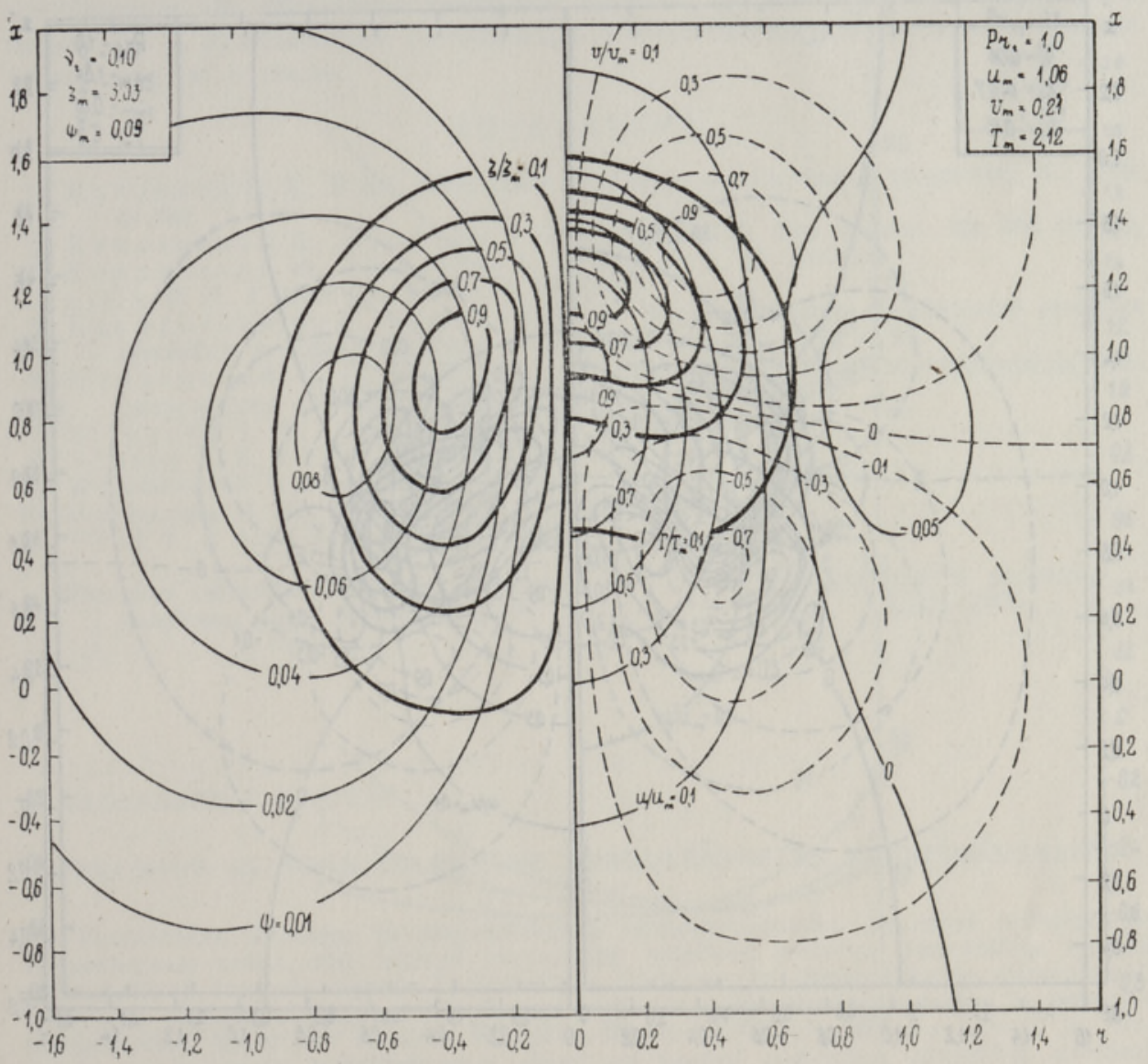

Рис. 5.

где $\tau=\frac{1}{A} \ln \left[t / t_{0}\right]$ и $A=4, B=0, C=3$ для динамического кольца и $A=2, B=2\left(\frac{\chi_{1}}{x \chi_{2}}\right), \quad C=3$ для конвективного кольца.

Уравнения (2), (3) и граничные условия (5) не изменяются по форме в результате преобразования координат. Условиями нормировки для системы уравнений в новых переменных являются (8).

\section{Результаты численного решения}

Расчеты проводились в пределах изменения $v_{t}=0,10-0,03$ и $\operatorname{Pr}_{t}=$ $=v_{t} / a_{t}=0,5-1,0$ для динамических колец и $v_{t}=0,10-0,01$ и $\operatorname{Pr}_{t}=$ $=0,5-1,0$ для конвективных колец. Во всех расчетах принималось $x=\chi_{1}=\chi_{2}=1$.

Проблемы выбора оптимальных размеров и положения области счета и задания распределений параметров на границах этой области рассмотрены в $\left[{ }^{3}\right]$, а подробное описание метода численного решения содержится в $\left[{ }^{6}\right]$.

На рис. 1, 2 представлены автомодельные распределения $\psi, \xi, u, v$ и $T$ в виде изолиний этих функций при $\operatorname{Pr}_{t}=1,0$ и $v_{t}=0,10$ и 0,03 соот- 


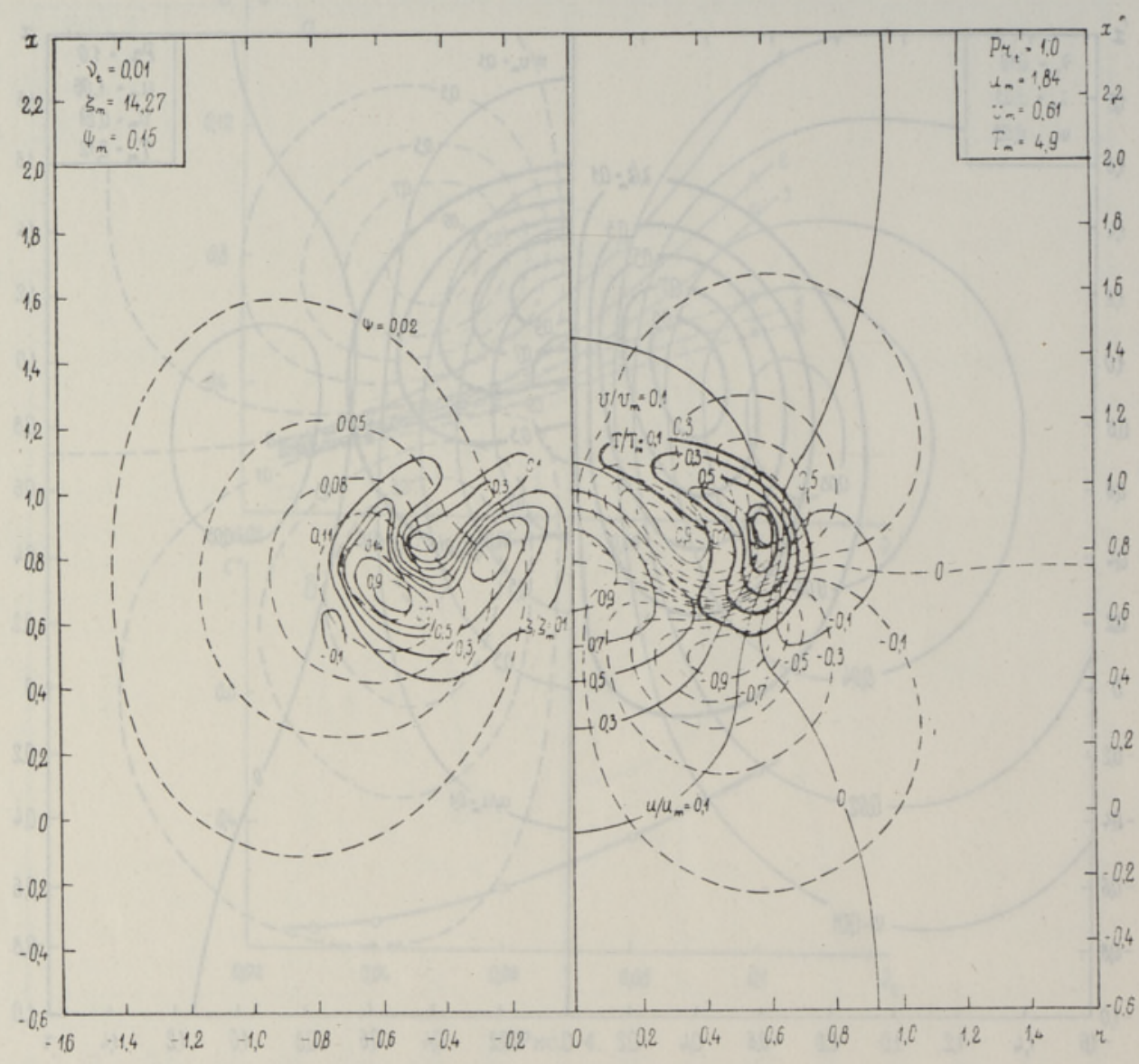

Рис. 6.

ветственно, а на рис. 3 распределения $T$ и $u$ при $v_{t}=0,06$ и $\operatorname{Pr}_{t}=0,5$ и 1,0 . Как явствует из этих графиков, основные качественные особенности полученных решений при разных значениях $v_{t}$ и $\mathrm{Pr}_{t}$ являются теми же, что и в случае плоского аналога вихревого кольца - вихревой пары $\left[{ }^{3,5}\right]$. В частности, при уменьшении $v_{t}$ наблюдается увеличение асимметрии распределения параметров кольца вдоль оси $x$ с вытягиванием зоны распространения завихренности в сторону, противоположную направлению основного движения кольца, и образованием периферийных максимумов температуры. Характерным для вихревых колец является бо́льшая степень влияния конвективных членов в уравнениях (12) и (13) по сравнению с вихревыми парами при тех же значениях $v_{t}$ и $\operatorname{Pr}_{t}$.

Графики скорости расширения динамических вихревых колец по характеристикам $c=r_{0} / x_{0}, c^{\prime}=r_{0,1} / x_{m}$ показаны на рис. $4, a$ (кривые $1-3$ и $4-6$ соответственно при $\left.\operatorname{Pr}_{t}=0,5 ; 0,75 ; 1,0\right)$ и по характеристике $c^{\prime \prime}=r_{0,1} / x_{0,1}$ на рис. $4, \sigma$. Можно констатировать, что расчетные и опытные [7] значения $c^{\prime \prime}$ в основном совпадают. Однако более подробного количественного сопоставления провести не удается из-за недостатка экспериментального материала по высокотурбулизированным вихревым кольцам динамического происхождения. 


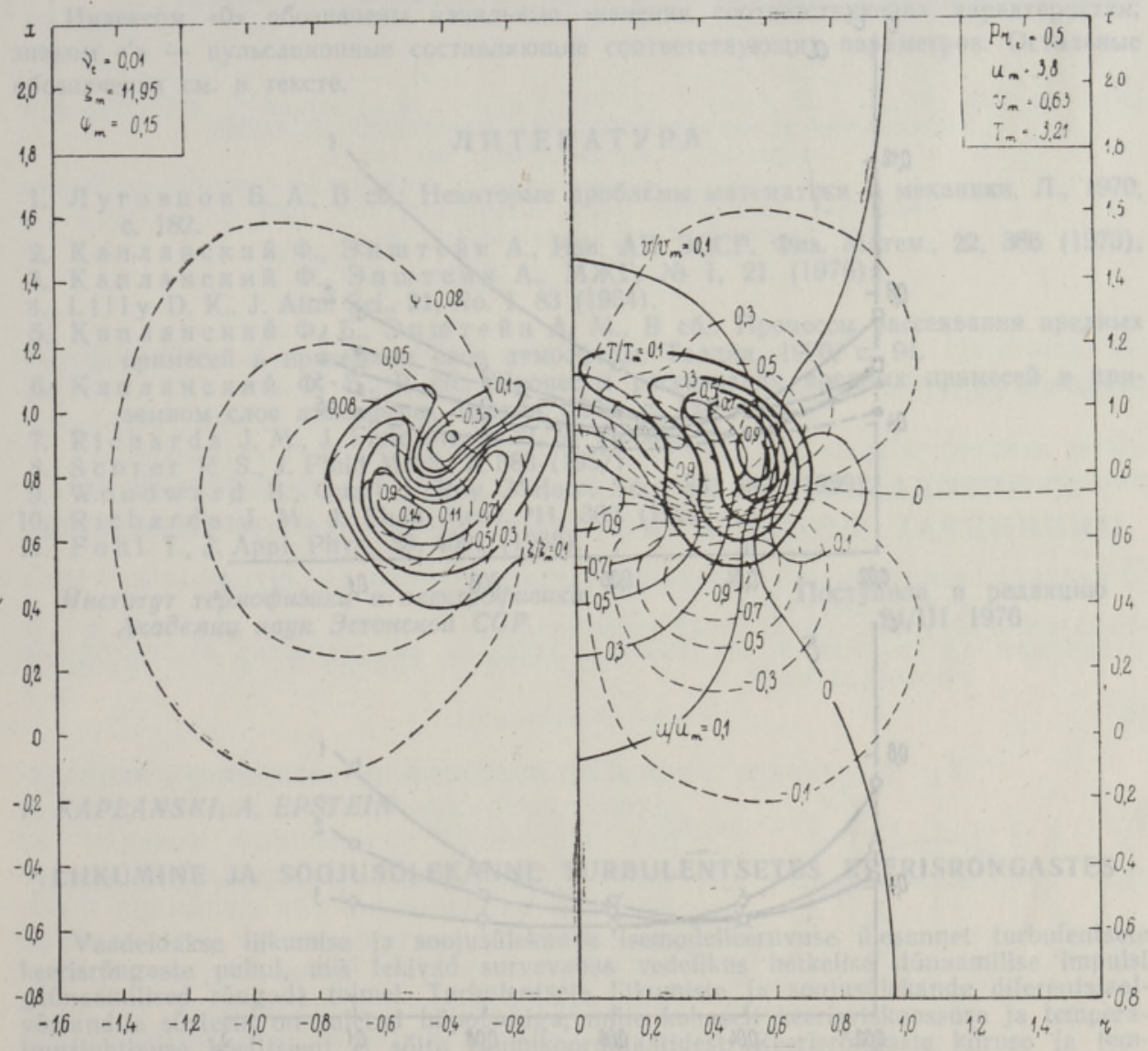

Puc. 7 .

Для конвективных вихревых колец распределения $\psi, \xi, u, v$ и $T$ изображены на рис. 5-7 для случаев $v_{t}=0,10$ и 0,01 при $\operatorname{Pr}_{t}=1,0$ и $v_{t}=0,01$ при $\operatorname{Pr}_{t}=0,5$ соответственно. Как видно, в качественном отношении они аналогичны тем же распределениям у конвективных вихревых пар $\left[{ }^{4,5}\right]$. В частности, при малых значениях $v_{t}$ отмечается ярко выраженная тенденция к образованию характерной кольцеобразной конфигурации конвективных элементов. Но в отличие от плоскопараллельного случая образование зоны с обратной по знаку завихренностью не сопровождается здесь противоположно направленной циркуляцией. Полученные в расчетах распределения основных параметров конвективных колец, в целом, находятся в качественном соответствии с имеющимися экспериментальными данными $\left[{ }^{8-10}\right]$.

Построенные на основании результатов численного решения графики характеристик расширения конвективных колец $c, c^{\prime}$ (кривые $1-3$ и 4 -6 соответственно на рис. $8, a$ при $\left.\operatorname{Pr}_{t}=0,5 ; 0,75 ; 1,0\right)$ и $c^{\prime \prime}$ (кривые $1-3$ на рис. 8 , б при тех же значениях $\operatorname{Pr}_{t}$ ) свидетельствуют о существовании минимума скорости расширения конвективного кольца. Однако значения коэффициента $c$, полученные в опытах $\left[{ }^{10,11}\right]$, существенно меньше минимальных по расчету. Причиной отмеченных выше расхождений является, возможно, недостаточная адекватность принятой 

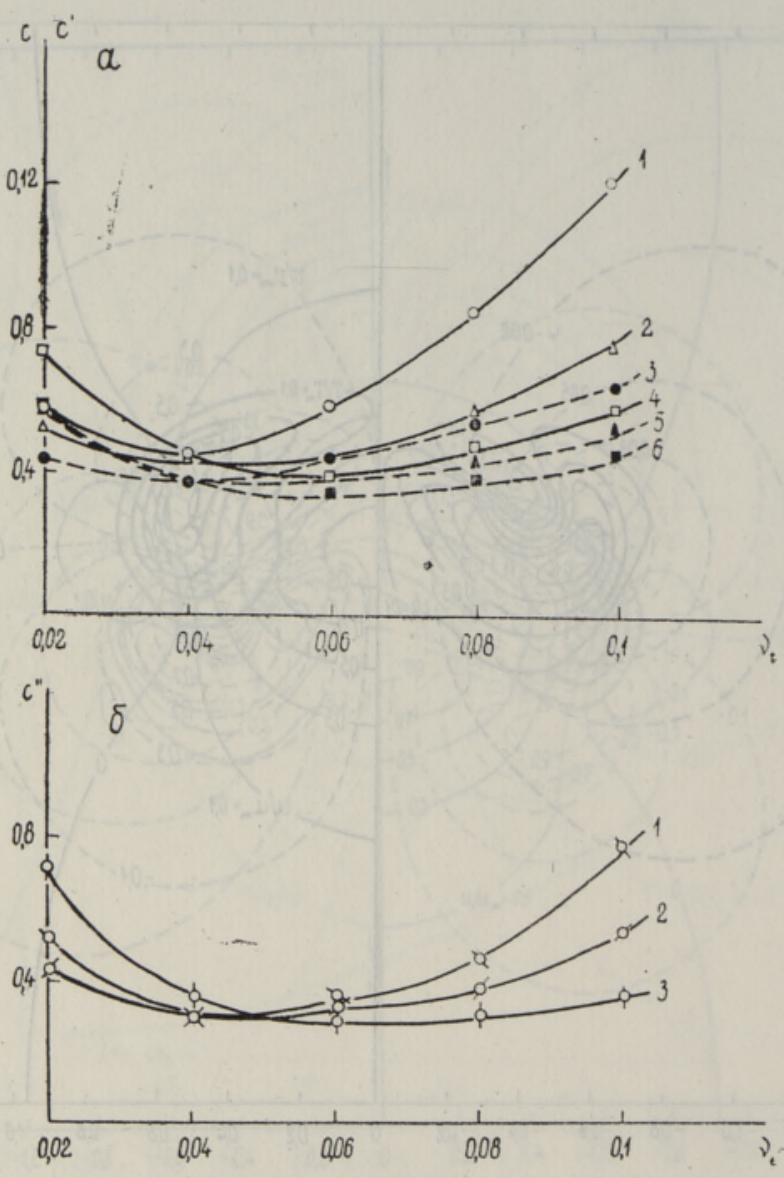

Рис. 8.

гипотезы о характере турбулентного переноса, описываемой уравнениями (7), применительно к течениям такого типа.

\section{О бозн ачения:}

$x, r$ - оси цилиндрической системы координат с началом в мгновенном точечном источнике ( $x-$ ось симметрии);

$u, v-$ составляющие осредненной скорости по направлениям $x, r$;

廿 $\quad-$ функция тока;

ร - вихрь;

$T$ - избыточная относительно окружающей среды температура;

$t \quad-$ время;

$v_{t}, Q_{t} \quad$ - турбулентные аналоги коэффициентов кинематической вязкости и температуропроводности;

$I, Q, Q_{t}$ - импульс, плавучесть и теплосодержание вихревого кольца соответственно;

$L, V, \Theta$ - характерные масштабы вихревого кольца (размер, скорость и температура);

$x_{0}, r_{0}-$ координаты точки, где $\zeta=\zeta_{m}\left(\zeta_{m}-\right.$ максимальное значение вихря);

$x_{0,1}, r_{0,1}$ - максимальная ордината и абсцисса изотермы $T=0,1 T_{m}\left(T_{m}-\right.$ максимальное значение температуры);

$x_{m} \quad$ - ордината точки $T=T_{m}$ на оси симметрии. 
Индексом «0» обозначены начальные значения соответствующих характеристик; знаком «'» - пульсационные составляющие соответствующих параметров. Остальные обозначения см. в тексте.

\section{Л И Т Е Р А Т Р А}

1. Л уго вц о в Б. А., В сб.: Некоторые проблемы математики и механики, Л., 1970, c. 182 .

2. К а пл а н ски й Ф., Эпш те й н А., Изв. АН ЭССР, Физ. Матем., 22, 386 (1973).

3. К а лл анский Ф., Эпштейн А., МЖГ, № 1, 21 (1976).

4. Li 11 y D. K., J. Atm. Sci., 21, No. 1, 83 (1964).

5. К а п л а с ки й Ф. Б., Эпш те й н А. М., В сб.: Процессы рассеивания вредных примесей в приземном слое атмосферы, Таллин, 1976, с. 91.

6. К а планский Ф. Б., В сб.: Процессы рассеивания вредных примесей в приземном слое атмосферы, Таллин, 1976, с. 124.

7. Ri ch a rd s J. M., J. Fluid Mech., 21, 97 (1965).

8. S c ore r R. S., J. Fluid Mech., 2, 583 (1957).

9. Wo o d w a r d B., Quart J. Roy. Meteor. Soc., 85, 583 (1959).

10. Rich ard s J. M., J. Fluid Mech., 11, 369 (1961).

11. F o h 1 T., J. Appl. Phys., 38, 4097 (1968).
Ннститут термофизики и электрофизики Академии наук Эстонской ССР
Поступила в редакцию $24 /$ III 1976

\section{F. KAPLANSKI, A. EPSTEIN}

\section{LIIKUMINE JA SOOJUSULEKANNE TURBULENTSETES KEERISRONGASTES}

Vaadeldakse liikumise ja soojusülekande isemodelleeruvuse ülesannet turbulentsete keerisrõngaste puhul, mis tekivad survevabas vedelikus hetkelise dünaamilise impulsi (dünaamilised rõngad) toimel. Turbulentsete liikumiste ja soojusülekande diferentsiaalvõrrandite süsteem on suletud hüpoteesiga, mille kohaselt keerisviskoossuse ja temperatuurijuhtivuse koefitsient ei sõltu ruumikoordinaatidest. Keerisrõngaste kiiruse ja temperatuuri jagunemise isemodelleeruv lahend on saadud nimetatud võrrandite arvulisel lahendamisel.

\section{F. KAPLANSKI, A. EPSTEIN}

\section{MOTION AND HEAT TRANSFER IN TURBULENT VORTEX RINGS}

A problem of the motion and heat transfer in turbulent vortex rings originating from an instantaneous source of impulse (dynamical rings) or buoyancy (convective rings) in the self-preserving stage is considered. The governing differential equations of turbulent motion and heat transfer are closed by means of the spatially constant eddy viscosity and heat transfer coefficients hypothesis. Distributions of vorticity, velocity and temperature of vortex rings are obtained from the numerical solution of the above-mentioned equations. 